\title{
Notes on Quasiminimality and Excellence
}

\author{
John T. Baldwin \\ Department of Mathematics, Statistics and Computer Science \\ University of Illinois at Chicago*
}

October 12, 2003

\begin{abstract}
Zilber's proposes [60] to prove 'canonicity results for pseudo-analytic' structures. Informally, 'canonical means the theory of the structure in a suitable possibly infinitary language (see Section 2) has one model in each uncountable power' while 'pseudoanalytic means the model of power $2^{\aleph_{0}}$ can be taken as a reduct of an expansion of the complex numbers by analytic functions'. This program interacts with two other lines of research. First is the general study of categoricity theorems in infinitary languages. After initial results by Keisler, reported in [31], this line was taken up in a long series of works by Shelah. We place Zilber's work in this context. The second direction stems from Hrushovski's construction of a counterexample to Zilber's conjecture that every strongly minimal set is 'trivial', 'vector space-like', or 'field-like'. This construction turns out to be very concrete example of the Abstract Elementary Classes which arose in Shelah's analysis. This paper examines the intertwining of these three themes.
\end{abstract}

The study of $(\mathcal{C},+, \cdot, \exp )$ leads one immediately to some extension of first order logic; the integers with all their arithmetic are first order definable in $(\mathcal{C},+, \cdot, \exp )$. Thus, the first order theory of complex exponentiation is horribly complicated; it is certainly unstable and so can't be first order categorical. One solution is to use infinitary logic to pin down the pathology. Insist that the kernel of the exponential map is fixed as a single copy of the integers while allowing the rest of the structure to grow. We describe in Section 5 Zilber's program to show, modulo certain (very serious) algebraic hypotheses, that $(\mathcal{C},+, \cdot, \exp )$ can be axiomatized by a categorical $L_{\omega_{1}, \omega}$-sentence.

Of course, the extension from first order logic causes the failure of the compactness theorem. (E.g., it is easy to write a sentence in $L_{\omega_{1}, \omega}$ whose only model is the natural numbers with successor). But there are some more subtle losses. In first order logic, a type can be given as a syntactic object - a consistent set of formulas. Consider the theory $T$ of a dense linear order without endpoints, a unary predicate $P(x)$ which is dense and codense, and an infinite set of constants arranged in order type $\omega+\omega^{*}$. Let $\boldsymbol{K}$ be class of all models of $T$ which omit the type of a pair of points, which are both in the cut determined by the constants. Now consider the types $p$ and $q$ which are satisfied by a point in the cut, which is in $P$ or in $\neg P$ respectively. Now $p$ and $q$ are each satisfiable in a member of $\boldsymbol{K}$ but they are not simultaneously satisfiable. This failure of amalgamation shows that a more subtle notion than consistency is needed to describe types.

We took 'canonical' above as meaning 'categorical in uncountable cardinalities'. The analysis of first order theories categorical in power is based on first studying strongly minimal sets: every definable subset is finite or cofinite. A natural generalization of this, particularly since it holds of simply defined subsets of $(\mathcal{C},+, \cdot, \exp )$, is to consider structures where every definable set is countable or cocountable. As we will see, the useful formulation of this notion requires some auxiliary homogeneity conditions. The role of homogeneity in studying categoricity in infinitary languages has been known for a long time. There is a rough translation between 'homogeneity'

\footnotetext{
*Partially supported by NSF grant DMS-0100594 and CDRF grant KM2-2246.
} 
hypotheses on a model and and corresponding 'amalgamation' hypotheses on the class of substructures of the model (Section 2). A structure is $\aleph_{1}$-homogeneous if for any two countable sequences $\boldsymbol{a}, \mathbf{b}$, which realize the same type, and any $c$, there is a $d$ such that $\boldsymbol{a} c$ and $\mathbf{b} d$ realize the same type. Thus, $\aleph_{1}$-homogeneity corresponds to amalgamation over arbitrary countable subsets. Keisler [31] proved the natural generalization of Morley's theorem for a sentence $\psi$ in $L_{\omega_{1}, \omega}$ modulo two assumptions:

1. Every model of $\psi$ has arbitrarily large elementary extensions.

2. Every model of $\psi$ is $\aleph_{1}$-homogeneous.

Neither of these assumptions has been eliminated. Marcus [36] gave an example of a minimal prime model with infinitely many indiscernibles and a modification by Shelah provides an example of a totally categorical sentence in $L_{\omega_{1}, \omega}$ which has no $\aleph_{1}$-homogeneous models. Shelah's notion of an excellent class (extremely roughly: 'amalgamation over (independent) $n$-dimensional cubes for all $n$ ' and ' $\aleph_{0}$-stability') provides a middle ground. An excellent class (See paragraph 2.0.9.) is a strengthening of Keisler's first assumption (provides not only arbitrarily large models but a certain control over their construction) while weakening the second to assert amalgamation only over certain configurations.

Recall the logic $L(Q)$ adds to first order logic the expression $(Q x) \phi(x)$ which holds if there are uncountably many solutions of $\phi$. I had asked whether an $\aleph_{1}$-categorical sentence in $L(Q)$ must have larger models and Shelah proved in [42] that an $\aleph_{1}$-categorical sentence in $L_{\omega_{1}, \omega}(Q)$ must have a model of power $\aleph_{2}$. There is a beautiful proof of this result in ZFC in [50]. Shelah has moved this kind of argument from $\left(\aleph_{1}, \aleph_{2}\right)$ to $\left(\lambda, \lambda^{+}\right)$in a number of contexts but getting arbitrarily large models just from categoricity in a single cardinal has remained intractable, although Shelah reported substantial but not yet written progress in the summer of 2003.

Shelah proved an analog to Morley's theorem in $[45,46]$ for 'excellent' classes defined in $L_{\omega_{1}, \omega}$. Assuming $2^{\aleph_{n}}<2^{\aleph_{n+1}}$, for all $n<\omega$, he also proved a stronger version of the following kind of converse: every sentence in $L_{\omega_{1}, \omega}$, which is categorical in $\aleph_{n}$, for all $n<\omega$ is excellent. The assumption of categoricity all the way up to $\aleph_{\omega}$ is shown to be essential in [18] by constructing for each $n$ a sentence $\psi_{n}$ of $L_{\omega_{1}, \omega}$ which is categorical up to $\aleph_{n}$ but has the maximal number of models in all sufficiently large cardinalities. He also asserted that these results 'should be reproved' for $L_{\omega_{1}, \omega}(Q)$. This 'reproving' has continued for 20 years and the finale is supposed to appear in the forthcoming Shelah $[47,48]$.

Zilber's approach is more analogous to the Baldwin-Lachlan (prime models over strongly minimal sets) approach than the Morley proof of the categoricity theorems. In fact, Zilber considers only the quasiminimal case, but a 'Baldwin-Lachlan' style proof was obtained by Lessmann for homogeneous model theory in [35] and for excellent classes in [34]. I.e., he proves every model is prime and minimal over a quasiminimal set.

We begin in Section 1 by recalling the basic notions of the Frä̈ssé construction and the notion of homogeneity. In Section 2, we sketch some results on the general theory of categoricity in non-elementary logics. In particular, we discuss both reductions to the 'first order logic with omitting types' and the 'syntax-free' approach of Abstract Elementary Classes. We turn to the development of the special case of quasiminimal theories in Section 3. This culminates in Zilber's first approximation of a quasiminimal axiomatization of complex exponentiation. In Section 4 we formulate the generalized Frä̈ssé construction and place it in the setting of Abstract Elementary Classes. We analyze this method for constructing first order categorical theories; we then see a variant to get examples in homogeneous model theory. Then we discuss the results and limitations of the program to obtain analytic representations of models obtained by this construction. Finally in Section 5 we return to Zilber's use of these techniques to study complex exponentiation. We describe the major algebraic innovations of his approach and the innovations to the Hrushovski construction which result in structures that are excellent but definitely not first order axiomatizable.

Many thanks to Rami Grossberg and Olivier Lessmann, who were invaluable in putting together this survey, but are not responsible for any errors. Comments by Assaf Hasson, David Kueker, Charles Steinhorn, Saharon 
Shelah, and Boris Zilber improved both the accuracy and the exposition.

\section{The Fraïssé Construction}

In the early 1950's Fraïssé [13] generalized Hausdorff's back and forth argument for the uniqueness of the rationals as countable dense linear order (without end points). He showed that any countable class $\boldsymbol{K}$ of finite relational structures closed under substructure and satisfying the joint embedding and amalgamation properties (see Definition 4.1.6) has a unique countable (ultra)-homogeneous member (denoted $\mathbb{G}$ ): any isomorphism between finite subsets of $\mathbb{G}$ extends to an automorphism. There are easy variants of this notion for locally finite classes in a language with function symbols. The existence of such structures is proved by iterating the amalgamation property and taking unions of chains. (See [21] for a full account.) Jónsson [28] extended the notion to arbitrary cardinals and Morley-Vaught [37] created an analogous notion for the class of models of first order theories with elementary embeddings as the morphisms. They characterized the homogeneous universal models in this situation as the saturated models. In general the existence of saturated models in power $\kappa$ requires that $\kappa=\kappa^{<\kappa}$ and $\kappa>2^{|L|}$; alternatively, one may assume the theory is stable. In particular, $\kappa$-saturated models are $\kappa$-homogeneous. Morley proved every uncountable model of a theory categorical in an uncountable power is saturated. Abstract versions of the Fraïssé construction undergird the next section; concrete versions dominate the last two sections of the paper.

\section{Syntax, Stability, Amalgamation}

This section is devoted to investigations of categoricity for non-elementary classes. We barely touch the immense literature in this area; see [15]. Rather we just describe some of the basic concepts and show how they arise from concrete questions of categoricity in $L_{\omega_{1}, \omega}$ and $L_{\omega_{1}, \omega}(Q)$. In particular, we show how different frameworks for studying nonelementary classes arise and some relations among them. Any serious study of this topic begins with $[30,31]$.

In its strongest form Morley's theorem asserts: Let $T$ be a first order theory having only infinite models. If $T$ is categorical in some uncountable cardinal then $T$ is complete and categorical in every uncountable cardinal. This strong form does not generalize to $L_{\omega_{1}, \omega}$; take the disjunction of a sentence which is categorical in all cardinalities with one that has models only up to, say, $\beth_{2}$. Since $L_{\omega_{1}, \omega}$ fails the upwards Löwenheim-Skolem theorem, the categoricity implies completeness argument that holds for first order sentences fails. However, if the $L_{\omega_{1}, \omega}$-sentence $\psi$ is categorical in $\kappa$, then, applying the downwards Löwenheim-Skolem theorem, for every sentence $\phi$ either $\psi \rightarrow \phi$ or all models of $\phi$ have cardinality less than $\kappa$. So if $\phi$ and $\psi$ are $\kappa$-categorical sentences with a common model of power $\kappa$ they are equivalent. We say a sentence of $L_{\omega_{1}, \omega}$ is complete if it either implies or contradicts every other $L_{\omega_{1}, \omega}$-sentence. Such a sentence is necessarily $\aleph_{0}$-categorical (using downward Löwenheim-Skolem). Moreover, every countable structure is characterized by a complete sentence its Scott sentence. So if a model satisfies a complete sentence, it is $L_{\infty, \omega}$-equivalent to a countable model. In particular, any model $M$ of $\psi$ is small : for every $n$ it realizes only countably many $L_{\omega_{1}, \omega}$-n-types (over the empty set). Moreover, if $\phi$ has a small model then $\phi$ is implied by a complete sentence satisfied in that model.

In the first order case it is trivial to reduce the study of categoricity to complete (for $L_{\omega, \omega}$ ) theories and theories share the fundamental properties of sentences- in particular, Löwenheim-Skolem down to $\aleph_{0}$. But an $L_{\omega_{1}, \omega^{-}}$ theory need not have a countable model. So while we want to reduce the categoricity problem to that for complete $L_{\omega_{1}, \omega}$-sentences, we cannot make the reduction trivially. We first show that if $\psi$ has arbitrarily large models and is categorical then $\psi$ extends to a complete sentence. A key observation is that if $\psi$ has arbitrarily large models then $\psi$ has models which realize few types. 
Lemma 2.0.1 Suppose $\psi$ has arbitrarily large models.

1. In every infinite cardinality $\psi$ has a model which realizes only countably many $L_{\omega_{1}, \omega}$-types over the empty set.

2. Thus, if $N$ is the unique model of $\psi$ in some cardinal, $\psi$ is implied by a consistent complete sentence $\psi^{\prime}$ which holds of $N$.

Proof. Since $\psi$ has arbitrarily large models we can construct a model with indiscernibles (Chapters 13-15 of [31]). Now take an Ehrenfeucht-Mostowski model $M$ for $\psi$ over a set of indiscernibles ordered by a $k$-transitive dense linear order. (These exist in every cardinal; take the order type of an ordered field.) Then for every $n, M$ has only countably many orbits of $n$-tuples and so realizes only countably many types in any logic where truth is preserved by automorphism - in particular in $L_{\omega_{1}, \omega}$. If $\psi$ is $\kappa$-categorical, let $\psi^{\prime}$ be the Scott sentence of this Ehrenfeucht-Mostowski model with cardinality $\kappa$.

If we don't assume $\psi$ has arbitrarily large models the reduction to complete sentences, sketched below, is more convoluted and uses hypotheses (slightly) beyond ZFC. In particular, the complete sentence $\psi^{\prime}$ does not hold, a priori of the categoricity model. The natural examples of $L_{\omega_{1}, \omega}$-sentences which have models of bounded cardinality (e.g. a linear order with a countable dense subset, or coding up an initial segment of the $V_{\alpha}$ hierarchy of all sets) have the maximal number of models in the largest cardinality where they have a model. Shelah discovers a dichotomy between such sentences and 'excellent' sentences. We expand on the notion of excellence at 2.0.9 and later in the paper. For the moment just think of the assertion that a complete $L_{\omega_{1}, \omega}$-sentence (equivalently, its class of models) is excellent as a step into paradise.

For any class $K$ of models, $I(\lambda, \boldsymbol{K})$ denotes the number of isomorphism types of members of $\boldsymbol{K}$, with cardinality $\lambda$. We may write $\psi$ instead of $\boldsymbol{K}$ if $\boldsymbol{K}$ is the class of models of $\psi$. We say that a class $\boldsymbol{K}$ has many models of cardinality $\aleph_{n}$ if $I\left(\aleph_{n}, \boldsymbol{K}\right) \geq \mu(n)$ (and few if not; there may not be any). We use as a black box the function $\mu(n)$ (defined precisely in [46]). Either GCH or $\neg O^{\#}$ imply $\mu(n)=2^{\aleph_{n}}$ but it is open whether it might be (consistently) smaller. The difficult heart of the argument is the following theorem of Shelah [45, 46]; we don't discuss the proof of this result but just show how this solution for complete sentences gives the result for arbitrary sentences of $L_{\omega_{1}, \omega}$.

Theorem 2.0.2 1. (For $n<\omega, 2^{\aleph_{n}}<2^{\aleph_{n+1}}$ ) A complete $L_{\omega_{1}, \omega}$-sentence which has few models in $\aleph_{n}$ for each $n<\omega$ is excellent.

2. (ZFC) An excellent class has models in every cardinality.

3. (ZFC)If $\phi$ is an excellent (see 2.0.9) $L_{\omega_{1}, \omega}$-sentence, $\phi$ is categorical in one uncountable cardinal $\kappa$ implies it is categorical in all uncountable cardinals.

So a nonexcellent class defined by a complete $L_{\omega_{1}, \omega}$-sentence. A complete $\psi$ may not have arbitrarily large models but, if not, it must have many models in some cardinal less than $\aleph_{\omega}$. Combining several results of Keisler, Shelah [45] shows:

Lemma 2.0.3 Assume $2^{\aleph_{0}}<2^{\aleph_{1}}$. Let $\psi$ be a sentence of $L_{\omega_{1}, \omega}$ that has at least one but less than $2^{\aleph_{1}}$ models of cardinality $\aleph_{1}$. Then $\psi$ has a small model of cardinality $\aleph_{1}$.

Proof. By Theorem 45 of [31], for any countable fragment $L^{*}$ containing $\psi$ and any $M \models \psi$ of cardinality $\aleph_{1}, M$ realizes only countably many $L^{*}$ types over the empty set. Theorem 2.2 of [42] says that if $\psi$ has a model of cardinality $\aleph_{1}$ which realizes only countably many types in each fragment then $\psi$ has a small model 
of cardinality $\aleph_{1}$. We sketch a proof of that theorem. Add to the language a linear order $<$, interpreted as a linear order $M$ with order type $\omega_{1}$. Using that $M$ realizes only countably many types in any fragment, write $L_{\omega_{1}, \omega}$ as a continuous increasing chain of fragments $L_{\alpha}$ such that each type in $L_{\alpha}$ realized in $M$ is a formula in $L_{\alpha+1}$. Add new $2 n+1$-ary predicates and $n+1$-ary functions $f_{n}$. Let $M$ satisfy $E_{n}(\alpha, \boldsymbol{a}, \mathbf{b})$ if and only if $\boldsymbol{a}$ and b realize the same $L_{\alpha}$-type and let $f_{n}$ map $M^{n+1}$ into the initial $\omega$ elements of the order, so that $E_{n}(\alpha, \boldsymbol{a}, \mathbf{b})$ implies $f_{n}(\alpha, \boldsymbol{a})=f_{n}(\alpha, \mathbf{b})$. Note: i) $E_{n}(\beta, \mathbf{y}, \mathbf{z})$ refines $E_{n}(\alpha, \mathbf{y}, \mathbf{z})$ if $\beta>\alpha$; ii) $E_{n}(0, \boldsymbol{a}, \mathbf{b})$ implies $\boldsymbol{a}$ and $\mathbf{b}$ satisfy the same quantifier free formulas; iii) if $\beta>\alpha, E_{n}(\beta, \boldsymbol{a}, \mathbf{b})$ implies $(\forall x)(\exists y) E_{n+1}(\alpha, x \boldsymbol{a}, y \mathbf{b})$. Thus, iv) for any $a \in M$ each equivalence relation $E_{n}(a, \mathbf{y}, \mathbf{z})$ has only countably many classes. All these assertions can be expressed by an $L_{\omega_{1}, \omega}$ sentence $\phi$. Now add a unary predicate symbol $P$ and a sentence $\chi$ which asserts $M$ is an end extension of $P(M)$. For every $\alpha<\omega_{1}$ there is a model $M_{\alpha}$ of $\phi \wedge \psi \wedge \chi$ with order type of $(P(M),<)$ greater than $\alpha$. (Start with $P$ as $\alpha$ and alternately take an elementary submodel for the smallest fragment $L^{*}$ containing $\phi \wedge \psi \wedge \chi$ and close down under <. After $\omega$ steps we have the $P$ for $M_{\alpha}$.) Now by Theorem 12 of [31] there is countable structure $\left(N_{0}, P\left(N_{0}\right)\right)$ such that $P\left(N_{0}\right)$ contains a copy of $(Q,<)$ and $N_{0}$ is an end extension of $P\left(N_{0}\right)$. By Theorem 28 of [31], $N_{0}$ has an $L^{*}$ elementary extension of cardinality $\aleph_{1}$. Fix an infinite decreasing sequence $d_{0}>d_{1}>\ldots$ in $N_{0}$. For each $n$, define $E_{n}^{+}(\mathbf{x}, \mathbf{y})$ if for some $i, E_{n}\left(d_{i}, \mathbf{x}, \mathbf{y}\right)$. Now using i), ii) and iii) prove by induction on the quantifier rank of $\phi$ that $N_{1} \models E_{n}^{+}(\boldsymbol{a}, \mathbf{b})$ implies $N_{1} \models \phi(\boldsymbol{a})$ if and only if $N_{1}=\phi(\mathbf{b})$ for every $L_{\omega_{1}, \omega}$ formula $\phi$. For each $n, E_{n}\left(d_{0}, \mathbf{x}, \mathbf{y}\right)$ refines $E_{n}^{+}(\mathbf{x}, \mathbf{y})$ and by iv) $E_{n}\left(d_{0}, \mathbf{x}, \mathbf{y}\right)$ has only countably many classes; so $N$ is small.

Using these two results, we easily derive a version of Morley's theorem for an $L_{\omega_{1}, \omega}$-sentence.

Theorem 2.0.4 Assume $2^{\aleph_{n}}<2^{\aleph_{n+1}}$ for $n<\omega$. If an $L_{\omega_{1}, \omega}$-sentence $\psi$ has an uncountable model then either

1. $\psi$ has many models in $\aleph_{n}$ for some $n<\omega$ or

2. $\psi$ has arbitrarily large models and $\psi$ is categorical in one uncountable cardinal $\kappa$ implies it is categorical all uncountable cardinals.

Proof. Suppose $\psi$ has few models in $\aleph_{n}$ for each $n<\omega$. By Lemma 2.0.3, choose a small model of $\psi$, say with Scott sentence $\psi^{\prime}$. Assuming $2^{\aleph_{n}}<2^{\aleph_{n+1}}$ for each $n$, Theorem 2.0.2 1) implies $\psi^{\prime}$ is excellent. By Theorem 2.0.2 2) $\psi^{\prime}$ and thus $\psi$ have arbitrarily large models. Now suppose $\psi$ is categorical in $\kappa>\aleph_{0}$. Then so is $\psi^{\prime}$ whence, by Theorem 2.0.2 3), $\psi^{\prime}$ is categorical in all uncountable powers.

To show $\psi$ is categorical above $\kappa$ note that by downward Löwenheim-Skolem all models of $\psi$ with cardinality at least $\kappa$ satisfy $\psi^{\prime}$; the result follows by the categoricity of $\psi^{\prime}$. If $\psi$ is not categorical in some cardinality $\mu<\kappa$, there must be a sentence $\theta$ which is inconsistent with $\psi^{\prime}$ but consistent with $\psi$. Applying the entire analysis to $\psi \wedge \theta$, we find a complete sentence $\psi^{\prime \prime}$ which has arbitrarily large models, is consistent with $\psi$ and contradicts $\psi^{\prime}$. But this is forbidden by categoricity in $\kappa$.

One corollary of this result is

Corollary 2.0.5 Assume $2^{\aleph_{0}}<2^{\aleph_{1}}$. If an $L_{\omega_{1}, \omega}$-sentence is categorical in $\aleph_{n}$ for $n<\omega$ then it is categorical in all cardinalities.

Hart and Shelah [18] have shown the necessity of the hypothesis of categoricity up to $\aleph_{\omega}$.

A key tool in the study of complete $L_{\omega_{1}, \omega}$-sentences is the reduction of the class of model of such sentences to classes which are 'closer' to being first order. We now give a full account of this easy reduction. Chang proved in [12] that the class of models of any sentence in $L_{\kappa^{+}, \omega}$ could be viewed as the class of reducts to $L$ of models of a first order theory in an expansion $L^{\prime}$ of $L$ which omitted a family of types. Chang (Lopez-Escobar [12]) used this observation to prove that the Hanf number for $L_{\kappa^{+}, \omega}$ is same as the Hanf number for omitting 
a family of $\kappa$ types. Shelah [42] took this reduction a step further and showed that the class of models of a complete sentence in $L_{\omega_{1}, \omega}$ are in 1-1 correspondence (mapping $L_{\infty, \omega}$-submodel to elementary submodel) with the class of atomic models of an appropriate first order theory in an expanded language. That is, to study the generalization of Morley's theorem to complete $L_{\omega_{1}, \omega}$-sentences it suffices to study classes of structures defined by a finite diagram, that is an $E C(T, \Gamma)$ class: those models of first order theory which omit all types from a specified collection $\Gamma$ of types in finitely many variables over the empty set. Abusing the $E C(T, \Gamma)$ notation, $E C(T$, Atomic) denotes the class of atomic models of $T$ (i.e. to conform to the notation we should write nonatomic). Most detailed study of the spectrum of $L_{\omega_{1}, \omega}$-sentences [42, 45, 46, 34, 16, 27] just work with finite diagrams (and usually under stronger homogeneity conditions).

Theorem 2.0.6 Let $\psi$ be a complete sentence in $L_{\omega_{1}, \omega}$. Then there is a countable language $L^{\prime}$ extending $L$ and a first order $L^{\prime}$-theory $T$ such that reduct is a 1-1 map from the atomic models of $T$ onto the models of $\psi$.

Proof. Let $L^{*}$ be a countable fragment of $L_{\omega_{1}, \omega}$ which contains all subformulas of $\psi$ and the conjunction of each $L_{\omega_{1}, \omega}$-type that is realized in a model of $\psi$. (This set is countable since complete sentences are small. Expand $L$ to $L^{\prime}$ by inductively adding a predicate $P_{\phi}(\mathbf{x})$ for each $L^{*}$-formula $\phi$. Fix a model of $\psi$ and expand it to an $L^{\prime}$-structure by interpreting the new predicates so that the new predicates represent each finite Boolean connective and quantification faithfully: E.g.

$$
P_{\neg \phi(\mathbf{x})} \leftrightarrow \neg P_{\phi}(\mathbf{x})
$$

and

$$
P_{(\forall \mathbf{x}) \phi(\mathbf{x})} \leftrightarrow(\forall \mathbf{x}) P_{\phi}(\mathbf{x}),
$$

and that, as far as first order logic can, the $P_{\phi}$ preserve the infinitary operations: for each $i$,

$$
P_{\bigwedge_{i} \phi_{i}(\mathbf{x})} \rightarrow P_{\phi_{i}(\mathbf{x})}
$$

Let $T$ be the first order theory of any such model and consider the set $\Gamma$ of types

$$
p_{\bigwedge_{i} \phi_{i}(\mathbf{x})}=\left\{\neg P_{\bigwedge_{i} \phi_{i}(\mathbf{x})}\right\} \cup\left\{P_{\phi_{i}(\mathbf{x})}: i<\omega\right\} .
$$

Note that if $q$ is an $L_{\omega_{1}, \omega}$-type realized in a model of $T, P_{\wedge q}$ generates a principal type in $T$. Now if $M$ is a model of $T$ which omits all the types in $\Gamma$ (in particular, if $M$ is an atomic model of $T$ ),$M \mid L \models \psi$ and each model of $\psi$ has a unique expansion to a model of $T$ which omits the types in $\Gamma$ (since this is an expansion by definitions in $\left.L_{\omega_{1}, \omega}\right)$.

So in particular, any complete sentence of $L_{\omega_{1}, \omega}$ can be replaced (for spectrum purposes) by considering the atomic models of a first order theory. Since all the new predicates are $L_{\omega_{1}, \omega}$-definable this is the natural extension of Morley's procedure of replacing each first order formula $\phi$ by a predicate symbol $P_{\phi}$, thus guaranteeing amalgamation over sets for first order categorical $T$; the amalgamation does not follow in this case. In general, finite diagrams do not satisfy the upper Löwenheim-Skolem theorem.

Remark 2.0.7 $\left(L_{\omega_{1}, \omega}(Q)\right)$ The situation for $L_{\omega_{1}, \omega}(Q)$ is more complicated. Some of the analysis of [45, 46] goes over directly. But many problems intervene and Shelah has devoted several articles (notably [49, 47, 48] to completing the analysis and a definitive version has not appeared. The difficulty in extending from $L_{\omega_{1}, \omega}$ to $L_{\omega_{1}, \omega}(Q)$ is in constructing models with the proper interpretation of the $Q$-quantifier. Following Keisler's analysis of this problem in [30] the technique is to consider various notions of strong submodel. Two notions are relevant: in the first, the relation of $M \prec K^{N}$ holds when definable sets which are intended to be 
countable $(M \models \neg(Q x) \phi(x))$ do not increase from $M$ to $N$. The seconds adds that definable sets intended to be uncountable $(M \models(Q x) \phi(x))$ increase from $M$ to $N$. The first notion gives an AEC; the second does not. The reduction $[50,47]$ is actually to an AEC along with the second relation as an auxiliary that guarantees the existence of standard models.

When Jónsson generalized the Fraïsse construction to uncountable cardinalities [28, 29], he did so by describing a collection of axioms, which might be satisfied by a class of models, that guaranteed the existence of a homogeneous-universal model; the substructure relation was an integral part of this description. Morley and Vaught [37] replaced substructure by elementary submodel and developed the notion of saturated model. Shelah $[50,51]$ generalized this approach in two ways. He moved the amalgamation property from a basic axiom to a constraint to be considered. (But this was a common practice in universal algebra as well.) He made the substructure notion a 'free variable' and introduced the notion of an Abstract Elementary Class: a class of structures and a 'strong' substructure relation which satisfied variants on Jonsson's axioms. To be precise

Definition 2.0.8 A class of L-structures, $(\boldsymbol{K}, \prec \boldsymbol{K})$, is said to be an abstract elementary class: AEC if both $\boldsymbol{K}$ and the binary relation $\prec \boldsymbol{K}$ are closed under isomorphism and satisfy the following conditions.

- A1. If $M \prec_{\boldsymbol{K}} N$ then $M \subseteq N$.

- A2. $\prec_{\boldsymbol{K}}$ is a partial order on $\boldsymbol{K}$.

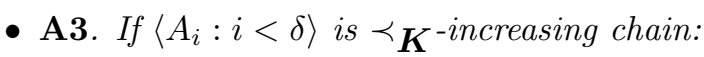

1. $\bigcup_{i<\delta} A_{i} \in \boldsymbol{K}$;

2. for each $j<\delta, A_{j} \prec \boldsymbol{K} \bigcup_{i<\delta} A_{i}$

3. if each $A_{i} \prec \boldsymbol{K} M \in \boldsymbol{K}$ then $\bigcup_{i<\delta} A_{i} \prec \boldsymbol{K} M$.

- A4. If $A, B, C \in \boldsymbol{K}, A \prec_{\boldsymbol{K}} C, B \prec_{\boldsymbol{K}} C$ and $A \subseteq B$ then $A \prec_{\boldsymbol{K}} B$.

- A5. There is a Löwenheim-Skolem number $\kappa(\boldsymbol{K})$ such that if $A \subseteq B \in K$ there is a $A^{\prime} \in \boldsymbol{K}$ with $A \subseteq A^{\prime} \prec \boldsymbol{K} B$ and $\left|A^{\prime}\right|<\kappa(\boldsymbol{K})$.

With $\prec \boldsymbol{K}$ as the notion of elementary submodel for such logics as first order logic, $L_{\omega_{1}, \omega}$, finite variable logic, classes defined in those logics become examples of AEC. Note that $L_{\omega_{1}, \omega}(Q)$ with the standard notion of elementary submodel is not an AEC (an uncountable union of countable sets can become uncountable). By interpreting $\prec \boldsymbol{K}$ in the manner described in Remark 2.0.7, sentences of $L_{\omega_{1}, \omega}(Q)$ define AEC's with Löwenheim number $\aleph_{0}$. The generalization to AEC is motivated by the fact that many arguments for the model theory of $L_{\omega_{1}, \omega}(Q)$ work as well in the abstract setting. We discuss a particularly relevant AEC for the Zilber program in Section 4.

By a very straightforward and short argument, Shelah shows in [50] that for every abstract elementary class $\boldsymbol{K}$ with vocabulary $L$, there is a vocabulary $L^{\prime}$ such that $K$ is the class of reducts to $L$ of $L^{\prime}$-structures which omit a certain set of types $\left(P C\left(T_{1}, L, \Gamma\right)\right)$. In particular, by the same argument as in [12] any AEC in a countable vocabulary with countable Lowenheim number which has a model of cardinality $\beth_{\left(2^{\omega}\right)^{+}}$has arbitrarily large models. Moreover, the same procedure allows the construction of Ehrenfreucht-Mostowski models and the deduction (modulo some amalgamation hypotheses) of stability from categoricity [47].

Note the following hierarchy of ease of definition where $A>B$ (read $>$ as 'is more general than') means every $B$ class is an $A$ class:

$$
P C\left(T_{1}, L, \Gamma\right)>A E C>L_{2^{{ }^{+}}, \omega}>E C(T, \Gamma)=\text { finite diagrams }>E C(T, \text { Atomic })>\text { first order } .
$$


The distinction given by the first inequality is very sharp. Silver (Chapter 18 of [31]) gives a simple example of a psuedoelementary class where the categoricity spectrum and its complement are both cofinal in the class of cardinals. But attempting to prove Morley's theorem for AEC (with additional hypotheses) is an active area.

Orthogonal to this syntactical hierarchy are various kinds of amalgamation hypotheses. There are a number of variants on homogeneity; here we mean the basic notion of sequential homogeneity. Perhaps the most important distinction is:

amalgamation over models $>$ homogeneity $=$ set amalgamation.

The original Keisler hypothesis for the categoricity theorem: that the model of power $\aleph_{1}$ is homogeneous led to an important topic, finite diagrams with amalgamation [41], i.e., an $E C(T, \Gamma)$ class with set amalgamation. This is the subject now called 'homogeneous model theory' (focusing on substructures of a large homogeneous model of the $E C(T, \Gamma)$-class); cf. [11] [17], [27]. Many of the results of stability and simplicity theory have been developed in this context. In particular, one can prove a stablility spectrum theorem very similar to the first order case. The elaborate development during the last 30 years of the model theory of Banach spaces is an example of homogeneous model theory (12.5 of [19]). More general than any of the classes discussed here is the study of classes where structures, which are amalgamation bases for extensions of the same cardinality, are dense [52, 53]. An early overview of all these questions is in the hard to locate Lazy Model Theorists guide [43].

Remark 2.0.9 Excellence: The notion of excellence as defined in [45] includes both an amalgamation component and a stability component. The idea arises from the attempt to construct arbitrarily large models. Vaught ([54]) proved that a theory with a countable atomic model $M_{0}$ has a model $M$ of power $\aleph_{1}$ : properly extend $M_{0}$ to $M_{1}$ (which is also atomic). Iterate, taking unions at limits. Shelah transferred the argument to convert a categorical model $M$ of size $\aleph_{1}$ to a model of size $\aleph_{2}$. The key idea is to analyze how every model can be built up from countable submodels. Using categoricity, the problem reduces to finding a proper elementary extension of $M$. For this, write $M$ as a chain of countable models $M_{i}$ and extend each $M_{i}$ to an $N_{i}$ The simplest way to guarantee $\bigcup_{i<\aleph_{1}} M_{i} \subset \bigcup_{i<\aleph_{1}} N_{i}$ is to guarantee that $N_{0} \cap M=M_{0}$, that is, that $M$ and $N_{0}$ are disjointly amalgamated over $M_{0}$. For this, some stability is used. To construct a model of power $\aleph_{3}$, from an $M$ of cardinality $\aleph_{2}$ the process is repeated. Now, after writing $M$ as a union of models of cardinality at most $\aleph_{1}$, one tries to again extend model by model. Now each model in the tower is decomposed into a chain of countable models. To reconstruct the tower one must amalgamate cubes of countable models and the system must be 'stable' to ensure the towers expand. Excellence asserts that a free $n$-dimensional cube of models can be completed. We have just sketched the use of excellence to build arbitrarily large models. In Section 3, we will discuss how in a restricted setting it produces uniqueness. Excellent classes have been explored by a number of authors. The 'main gap' was carried over from first order logic to excellent classes by Grossberg and Hart [16]; Lessmann [34] expounds the categoricity situation, explaining the distinction between homogeneous and excellent categorical classes.

\section{Quasiminimality and Excellence}

In the first subsection we define (a slight variant of) Zilber's notion of a quasiminimal excellent class and sketch his proof that quasiminimal excellent classes are categorical in all uncountable powers. A quasiminimal excellent class is a class of structures such that each structure admits a combinatorial geometry and certain amalgamations over free configurations for this geometry are insured. In the second subsection, we describe a quasiminimal excellent approximation of complex exponentiation [57]. 


\subsection{Abstract Quasiminimality}

A class $\boldsymbol{K}$ is quasiminimal excellent [60] if it satisfies the following four conditions. We speak of abstract quasiminimality because the notion is defined here in terms of an unspecified combinatorial geometry. A specific application might, for example, define $a \in \operatorname{cl}(A)$ if $a$ is in a countable set that is $L_{\omega_{1}, \omega}$-definable with parameters from $A$. This notion has a fundamental difficulty: in general, one may have elementarily equivalent sets $X$ and $Y$ whose closures are not isomorphic. This anomaly is avoided for the ordinary notion of algebraic closure by minimizing the size of the finite set witnessing $a \in \operatorname{acl}(X)$; the notion of excellence is the substitute here. Quasiminimal excellence is to Shelah's notion of excellence as strongly minimal sets are to the study of $\omega$-stable first order theories.

Assumption 3.1.1 (Condition I) Let $\boldsymbol{K}$ be a class of L-structures which admit a monotone idempotent closure operation cl taking subsets of $M \in \boldsymbol{K}$ to substructures of $M$ such that $\mathrm{cl}$ has finite character.

Let $G$ be a subset of $H, H^{\prime}$ and all three be in $\boldsymbol{K}$. A map from $X \subset H-G$ to $X^{\prime} \subset H^{\prime}-G$ is called a partial $G$-monomorphism if its union with the identity map on $G$ preserves quantifier free formulas.

Assumption 3.1.2 (Condition II) Let $G \subseteq H, H^{\prime} \in \boldsymbol{K}$ with $G$ empty or in $\boldsymbol{K}$.

1. If $f$ is a bijection between $X$ and $X^{\prime}$ which are separately cl-independent (over $G$ ) subsets of $H$ and $H^{\prime}$ then $f$ is a partial $G$-monomorphism.

2. If $f$ is a partial $G$-monomorphism from $H$ to $H^{\prime}$ with finite domain $X$ then for any $y \in H$ there is $y^{\prime} \in H^{\prime}$ such that $f \cup\left\{\left\langle y, y^{\prime}\right\rangle\right\}$ extends $f$ to a partial $G$-monomorphism.

3. If $f$ is a partial G-monomorphism from $H$ to $H^{\prime}$ taking $X \cup\{y\}$ to $X^{\prime} \cup\left\{y^{\prime}\right\}$ then $y \in \operatorname{cl}(X G)$ iff $y^{\prime} \in \operatorname{cl}\left(X^{\prime} G\right)$.

Condition 3) has an a priori unlikely strength: quantifier free formulas determine the closure; in practice, the language is specifically expanded to guarantee this condition. Part 2 of Assumption 3.1.2 implies that each $M$ with $G \subseteq M \in \boldsymbol{K}$ is finite sequence homogeneous over $G$.

In the following definition it is essential that $\subset$ be understood as proper subset.

\section{Definition 3.1.3 1. For any $Y, \operatorname{cl}^{-}(Y)=\bigcup_{X \subset Y} \operatorname{cl}(X)$.}

2. We call $C$ (the union of) an $n$-dimensional cl-independent system if $C=\mathrm{cl}^{-}(Z)$ and $Z$ is an independent set of cardinality $n$.

To visualize a 3 -dimensional independent system think of a cube with the empty set at one corner $A$ and each of the independent elements $z_{0}, z_{1}, z_{2}$ at the corners connected to $A$. Then each of $\operatorname{cl}\left(z_{i}, z_{j}\right)$ for $i<j<3$ determines a side of the cube: $\mathrm{cl}^{-}(Z)$ is the union of these three sides; $\mathrm{cl}(Z)$ is the entire cube.

Assumption 3.1.4 (Condition III) Let $G \subseteq H, H^{\prime} \in \boldsymbol{K}$ with $G$ empty or in $\boldsymbol{K}$. Suppose $Z \subset H-G$ is an n-dimensional independent system, $C=\mathrm{cl}^{-}(Z)$, and $X$ is a finite subset of $\operatorname{cl}(Z)$. Then there is a finite $C_{0}$ contained in $C$ such that: for every $G$-partial monomorphism $f$ mapping $X$ into $H^{\prime}$, for every $G$-partial monomorphism $f_{1}$ mapping $C$ into $H^{\prime}$, if $f \cup\left(f_{1}\left\lceil C_{0}\right)\right.$ is a G-partial monomorphism, $f \cup f_{1}$ is also a G-partial monomorphism. 
Thus Condition III, which is the central point of excellence, asserts (e.g. in dimension 3) that the type of any element in the cube over the union of the three given sides is determined by the type over a finite subset of the sides. The 'thumbtack lemma' of Subsection 3.2 verifies this condition in a specific algebraic context.

Assumption 3.1.5 (Condition IV) cl satisfies the exchange axiom: $y \in \operatorname{cl}(X x)-\operatorname{cl}(X)$ implies $x \in \operatorname{cl}(X y)$.

Zilber omits exchange in the fundamental definition but it arises in the natural contexts he considers so we make it part of quasiminimal excellence. Note however that in Section 4, the examples of first order theories with finite Morley rank greater than 1 (the parameter $\alpha$ of the construction is greater than 1) fail exchange. We say a closure operation satisfies the countable closure condition if the closure of a countable set is countable. We easily see:

Lemma 3.1.6 Suppose Assumptions I and II are satisfied by $\mathrm{cl}$ on an uncountable structure $M$ and satisfies the countable closure condition.

1. For any finite set $X \subseteq H \in \boldsymbol{K}$, if $a, b \in H-\operatorname{cl}(X)$, a,b realize the same $L_{\omega_{1}, \omega}$ type over $X$.

2. For every uncountable $M \in \boldsymbol{K}$, every $L_{\omega_{1}, \omega}$ definable set is countable or cocountable. This implies that $a \in \operatorname{cl}(X)$ iff it satisfies some $\phi$ over $X$, which has only countably many solutions.

Proof. Condition 1) follows directly from 1) and 2) of Assumption 2 by constructing a back and forth. To see condition 2), suppose both $\phi$ and $\neg \phi$ had uncountably many solutions with $\phi$ defined over $X$. Then there are $a$ and $b$ satisfying $\phi$ and $\neg \phi$ respectively and neither is in $\operatorname{cl}(X)$; this contradicts 1$)$.

The $\omega$-homogenity yields by an easy induction:

Lemma 3.1.7 Suppose Assumptions I and II hold. If $\operatorname{cl}(X)$ and $\operatorname{cl}(Y)$ are countable and $X$ is independent then any isomorphism between $X$ and $Y$ extends to an isomorphism of $\operatorname{cl}(X)$ and $\operatorname{cl}(Y)$

For algebraic closure the countability restriction is unnecessary. We now use Assumption 3.1.4 to remove the restriction in excellent classes.

Theorem 3.1.8 Let $\boldsymbol{K}$ be a quasiminimal excellent class and suppose $H, H^{\prime} \in \boldsymbol{K}$ satisfy the countable closure condition. Let $\mathcal{A}, \mathcal{A}^{\prime}$ be cl-independent subsets of $H, H^{\prime}$ with $\operatorname{cl}(\mathcal{A})=H, \operatorname{cl}\left(\mathcal{A}^{\prime}\right)=H^{\prime}$, respectively, and $\psi$ a bijection between $\mathcal{A}$ and $\mathcal{A}^{\prime}$. Then $\psi$ extends to an isomorphism of $H$ and $H^{\prime}$.

Thus $\boldsymbol{K}$ has a unique model with countable closures in each uncountable cardinality.

We sketch the proof of Theorem 3.1.8. Fix a countable subset $\mathcal{A}_{0}$ of $\mathcal{A}$; without loss of generality, we can assume $\psi$ is the identity on $\mathcal{A}_{0}$ and work over $G=\operatorname{cl}\left(\mathcal{A}_{0}\right)$. So from now on monomorphism means monomorphism over $G$ and $\operatorname{cl}(X)$ means $\operatorname{cl}\left(\mathcal{A}_{0} X\right)$.

Note that $\psi$ is a monomorphism and so is $\psi_{0}=\psi \mid \mathcal{A}_{0}$. By Lemma 3.1.7 and induction, for any independent $X$ with $|X| \leq \aleph_{0}, \psi \mid X$ extends to a isomorphism from $\operatorname{cl}(X)$ to $\operatorname{cl}(\psi(X))$. Taking unions of an increasing chain, we can even assume $|X|=\aleph_{1}$.

Note also that $H=\lim _{X \subset \mathcal{A} ;|X|<\aleph_{0}} \operatorname{cl}(X)$. We have the obvious directed system on $\left\{\operatorname{cl}(X): X \subset \mathcal{A} ;|X|<\aleph_{0}\right\}$. So the theorem follows immediately if for each finite $X$ we can choose $\psi_{X}: \operatorname{cl}(X) \rightarrow H^{\prime}$ so that $X \subset Y$ implies $\psi_{X} \subset \psi_{Y}$. We prove this by induction on $|X|$. Suppose $|Y|=n+1$ and we have appropriate $\psi_{X}$ for $|X|<n+1$. We will prove two statements by induction. 
1. $\psi_{Y}^{-}: \mathrm{cl}^{-}(Y) \rightarrow H^{\prime}$ defined by $\psi_{Y}^{-}=\bigcup_{X \subset Y} \psi_{X}$ is a monomorphism.

2. $\psi_{Y}^{-}$extends to $\psi_{Y}$ defined on $\operatorname{cl}(Y)$.

The first step is done by induction and $\omega$-homogeneity using Lemma 3.1.7. The exchange axiom is used to guarantee that the maps $\psi_{Y}^{\prime}$ for $Y^{\prime} \supset Y$ agree where more than one is defined. The second is follows by Assumption 3.1.4 and induction using Lemma 3.1.7 and the fact that $\operatorname{cl}(Y)$ is countable. We have shown that the isomorphism type of a structure in $\boldsymbol{K}$ is determined by the cardinality of a basis for the geometry. If the $\boldsymbol{K}$ satisfies the countable closure condition, for uncountable models, the size of a model is the same as its dimension so we get categoricity.

A natural way to require countable closure condition of all members of $\boldsymbol{K}$ is to axiomatize the class in $L_{\omega_{1}, \omega}(Q)$; for the next example $L_{\omega_{1}, \omega}$ suffices because of a clever choice of the closure relation.

\subsection{Covers of the multiplicative group of $\mathbb{C}$}

The first approximation to a quasiminimal axiomatization of complex exponentiation considers short exact sequences of the following form.

$$
0 \rightarrow Z \rightarrow H \rightarrow F^{*} \rightarrow 0
$$

$H$ is a torsion-free divisible abelian group (written additively), $F$ is an algebraically closed field, and exp is the homomorphism from $(H,+)$ to $\left(F^{*}, \cdot\right)$, the multiplicative group of $F$. We can code this sequence as a structure:

$$
(H,+, E, S)
$$

where $E\left(h_{1}, h_{2}\right)$ iff $\exp \left(h_{1}\right)=\exp \left(h_{2}\right)$ and we pull back sum by the defining $H \models S\left(h_{1}, h_{2}, h_{3}\right)$ iff $F \models$ $\exp \left(h_{1}\right)+\exp \left(h_{2}\right)=\exp \left(h_{3}\right)$. Thus $H$ now represents both the multiplicative and additive structure of $F$.

Lemma 3.2.1 There is an $L_{\omega_{1}, \omega}$-sentence $\Sigma$ such that there is a 1-1 correspondence between models of $\Sigma$ and sequences (1).

The sentence asserts first that the quotient of $H$ by $E$ with + corresponding to $\times$ and $S$ to + is an algebraically closed field. We use $L_{\omega_{1}, \omega}$ to guarantee the kernel is 1-generated. This same proviso insures that the relevant closure condition has countable closures. Now the key result asserts.

Theorem 3.2.2 For an appropriate definition of closure, $\Sigma$ is quasiminimal excellent with the countable closure condition and categorical in all uncountable powers.

In this context the appropriate cl on the domain $H$ of a model of $\Sigma$ is defined by

$$
\operatorname{cl}(X)=\exp ^{-1}(\operatorname{acl}(\exp (X))
$$

where acl is the field algebraic closure in $F$. It is easy to check that cl gives a combinatorial geometry such that the countable closure of countable sets is countable. (Strictly speaking, the language will have to be expanded to guarantee Assumption 3.1.2.3.) The main algebraic ingredient in this argument arises from the treatment of the divisible closure (in the multiplicative group of the field). 
Definition 3.2.3 By a divisibly closed multiplicative subgroup associated with $a \in \mathbb{C}^{*}, a^{\mathbb{Q}}$, we mean a choice of a multiplicative subgroup containing a and isomorphic to the group $(\mathbb{Q},+)$.

Definition 3.2.4 We say $b_{1}^{\frac{1}{m}} \in b_{1}^{\mathbb{Q}}, \ldots b_{\ell}^{\frac{1}{m}} \in b_{\ell}^{\mathbb{Q}} \subset \mathbb{C}^{*}$, determine the isomorphism type of $b_{1}^{\mathbb{Q}}, \ldots b_{\ell}^{\mathbb{Q}} \subset \mathbb{C}^{*}$ over the subfield $k$ of $\mathbb{C}$ if given subgroups of the form $c_{1}^{\mathbb{Q}}, \ldots c_{\ell}^{\mathbb{Q}} \subset \mathbb{C}^{*}$ and $\phi_{m}$ such that

$$
\phi_{m}: k\left(b_{1}^{\frac{1}{m}} \ldots b_{\ell}^{\frac{1}{m}}\right) \rightarrow k\left(c_{1}^{\frac{1}{m}} \ldots c_{\ell}^{\frac{1}{m}}\right)
$$

is a field isomorphism it extends to

$$
\phi_{\infty}: k\left(b_{1}^{\mathbb{Q}}, \ldots b_{\ell}^{\mathbb{Q}}\right) \rightarrow k\left(c_{1}^{\mathbb{Q}}, \ldots c_{\ell}^{\mathbb{Q}}\right)
$$

In the following, $\sqrt{ } 1$ denotes the subgroup of roots of unity. We call this result the thumbtack lemma based on the following visualization of Kitty Holland. The various $n t h$ roots of $b_{1}, \ldots b_{m}$ hang on threads from the $b_{i}$. These threads can get tangled; but the theorem asserts that by sticking in a finite number of thumbtacks one can ensure that the rest of strings fall freely. The proof involves the theory of fractional ideals of number fields, Weil divisors, and the normalization theorem. For $a_{1}, \ldots a_{r}$ in $\mathbb{C}$, we write $\operatorname{gp}\left(a_{1}, \ldots a_{r}\right)$ for the multiplicative subgroup generated by $a_{1}, \ldots a_{r}$. The following general version of the theorem is applied for various sets of parameters to prove quasiminimal excellence.

\section{Theorem 3.2.5 (thumbtack lemma) [57]}

Let $P \subset \mathbb{C}$ be a finitely generated extension of $\mathbb{Q}$ and $L_{1}, \ldots L_{n}$ algebraically closed subfields of the algebraic closure of P. Fix multiplicatively divisible subgroups $a_{1}^{\mathbb{Q}}, \ldots a_{r}^{\mathbb{Q}}$ with $a_{1}, \ldots a_{r} \in P^{*}$ and $b_{1}^{\mathbb{Q}}, \ldots b_{\ell}^{\mathbb{Q}} \subset \mathbb{C}^{*}$. If $b_{1} \ldots b_{\ell}$ are multiplicatively independent over $\operatorname{gp}\left(a_{1}, \ldots a_{r}\right) \cdot \sqrt{ } 1 \cdot L_{1}^{*} \cdot \ldots L_{n}^{*}$ then for some $m b_{1}^{\frac{1}{m}} \in b_{1}^{\mathbb{Q}}, \ldots b_{\ell}^{\frac{1}{m}} \in$ $b_{\ell}^{\mathbb{Q}} \subset \mathbb{C}^{*}$, determine the isomorphism type of $b_{1}^{\mathbb{Q}}, \ldots b_{\ell}^{\mathbb{Q}}$ over $P\left(L_{1}, \ldots L_{n}, \sqrt{ } 1, a_{1}^{\mathbb{Q}}, \ldots a_{r}^{\mathbb{Q}}\right)$.

We describe these notions in terms of formulas rather than elements.

Definition 3.2.6 $\quad$ 1. Let $V$ be an irreducible variety over $C \subseteq F$. The sequence associated with $V$ over $C$ is a sequence

$$
\left\{V^{\frac{1}{m}}: m \in \omega\right\}
$$

such that $V^{1}=V$ and for any $m, n \in \omega$, raising to the mth power maps $V^{\frac{1}{n m}}$ to $V^{\frac{1}{n}}$.

2. If $V^{\prime} \subseteq V$ are varieties in $n$-variables over $C$, the pair

$$
\tau=\left(V-V^{\prime},\left\{V^{\frac{1}{m}}: m \in \omega\right\}\right)
$$

is called an almost finite $n$-type over $C$.

3. Zilber calls a principal type given by a difference of varieties $V-V^{\prime}$ a finite $n$-type over $C$.

One of the key ideas discovered by Shelah in the investigation of non-elementary classes is that in order for types to be well-behaved one may have to make restrictions on the domain. (E.g., we may be able to amalgamate types over models but not arbitrary types.) This principle is illustrated by the following definition and result of Zilber. 
Definition 3.2.7 $C \subseteq F$ is finitary if $C$ is the union of the divisible closure (in $\mathbb{C}^{*}$ ) of a finite set and finitely many algebraically closed fields.

To prove the following result, apply the thumbtack lemma with the $L_{i}$ as the fields and the $\boldsymbol{a}_{i}$ as the finite set.

Corollary 3.2.8 Any almost finite n-type over a finitary set is a finite n-type.

Sketch of Proof of Theorem 3.2.2. Another application of the thumbtack lemma gives directly the homogeneity conditions of Assumption 3.1.2. Exchange, Assumption 3.1.5, is immediate from the definition of closure (3.2). Finitary sets are more general than the $n$-dimensional independent systems in the definition of quasiminimal excellence, since the subsets don't have to be independent. So if $X$ is a sequence associated with a variety $V$ over an $n$-dimensional independent system $C$, applying the thumbtack lemma again allows us to reduce $X$ to a formula over a finite set yielding Assumption 3.1.4. So we finish by Theorem 3.1.8. $\quad \square_{3.2 .2}$

We have shown the expansion of the complex numbers by naming the congruence (on the additive group) induced by exponentiation is quasiminimal excellent. This argument is rather ad hoc; one just checks the property of quasi-minimal excellence with no specific model theoretic innovations in the argument. In the next section we see a family of constructions for quasiminimal excellent classes.

\section{The Generalized Fraïssé construction}

In the 1950's Fraïssé generalized the Cantor-Hausdorff proof of the uniqueness of countable dense linear orders (without endpoints) by showing a class of finite relational structures that has the amalgamation property over arbitrary substructures gives rise to a countable homogeneous structure. This construction was generalized to uncountable cardinals by Jónnson and inspired the Morley-Vaught invention of saturated models. Shelah generalized the notion still further with various approximations to his notion of an abstract elementary class; key to this generalization is replacing the concrete notion of substructure by a 'strong submodel' notion which is described axiomatically. Although the Fraïssé models were $\aleph_{0}$-categorical, all but the most trivial were inherently unstable. Hrushovski [25, 20] constructs stable examples by defining a notion of strong submodel in terms of a function mapping finitely generated structures into discrete subgroups of the reals (or into the integers).

\subsection{Weak Ranks and Strong Submodels}

We reprise the general construction in the form we described in [3]. Many explicit examples are discussed in that paper. Let $\langle K(N), \wedge, \vee\rangle$ be a lattice of substructures of a model $N$. For purposes of this paper a rank is a function $\delta$ from $K(N)$ to a discrete subgroup of the reals that is defined on each $N$ in a class $\boldsymbol{K}$. This notion of rank is much weaker than any other rank notion used in stability theory. We write $\delta(A / B)=\delta(A \vee B)-\delta(B)$ to indicate the relativization of the rank. We demand only that $\delta$ is monotonic: if $B \subseteq A, C \subseteq N$ and $A \wedge C=B$,

$$
\delta(A / B) \geq \delta(A / C)
$$

This requirement can be rephrased as asserting that $\delta$ is lower semimodular: for any $A, B$,

$$
\delta(A \vee C)-\delta(C) \leq \delta(A)-\delta(A \wedge C) .
$$

We say $\delta$ is modular if the inequality is an equality. Examples of $\delta$ include cardinality, relation size (number of instances of a relation), vector space dimension, and transcendence degree. All of these but the last are 
modular. The simplest example of 'relation size' is just the number of edges in a (symmetric) graph. As in [58] we say the rank is a predimension when the range of $\delta$ is the integers. There are many variants of this construction. Each depends on the choice of a class $\boldsymbol{K}$ and a rank function on members of $\boldsymbol{K}$. Many of the ranks are obtained by standard combinations of ones that are already known. If $\delta_{1}, \delta_{2}$ are ranks defined on a class $\boldsymbol{K}$, so are

$$
\delta=\alpha \delta_{1}+\beta \delta_{2}
$$

for any positive reals $\alpha, \beta$ and

$$
\delta=\alpha \delta_{1}-\beta \delta_{2}
$$

for any positive reals $\alpha, \beta$ if $\delta_{2}$ is modular! With this observation, most of the examples of this construction can be seen as built up from the examples in the previous paragraph. Irrational $\alpha, \beta$ correspond to the construction of strictly stable structures $[10,9,20]$ and will play no further role here.

Example 4.1.1 Let $\delta=\alpha \delta_{1}-\beta \delta_{2}$.

1. The class $(\boldsymbol{K}, \delta)$ is called ab initio if $\boldsymbol{K}$ is a universal class of relational structures, $\delta_{1}$ is cardinality and $\delta_{2}$ is the number of relations. This gives rise to the new strongly minimal set $(\alpha=\beta=1$ ) [25], a non-Desarguesian projective plane $(\alpha=2, \beta=1)$ [5], a strictly stable $\aleph_{0}$-categorical theory (Hrushovski $\alpha$ irrational, $\beta=1$ ), and theories with infinite weight (sequence of irrational $\alpha$ ) [20] and almost sure theorey of graphs with edge probability $n^{-\alpha}$ ( $\alpha$ irrational, $\beta=1$ but different $\boldsymbol{K}$ ) [9, 10].

2. Bicolored fields $\left(\alpha=k, \beta=1\right.$ ) [39, 7, 8] are expansions of a field by a unary predicate; $\delta_{1}=d_{f}$ is transcendence degree; $\delta_{2}$ counts the number of points in $P$.

Let $T_{-1}$ be a first order theory such that any subset $X$ of a model $N$ of $T_{-1}$ is contained in a minimal submodel of $N$; this implies there is a natural notion of a finitely generated model. We denote this submodel $\langle X\rangle_{N}$, dropping the subscript $N$ when the choice of $N$ is evident. This condition is clearly satisfied if $T_{-1}$ is universally axiomatized or strongly minimal and almost all of our examples fall into one of these two classes. Let $\overline{\boldsymbol{K}}_{-1}=\bmod \left(T_{-1}\right) ; \boldsymbol{K}_{-1}$ is the finitely generated members of $\overline{\boldsymbol{K}}_{-1}$.

The construction of the homogeneous model is made with respect to a notion of strong substructure.

Definition 4.1.2 1. For $A, B \in \overline{\boldsymbol{K}}_{-1}$, we say $A$ is a strong substructure of $B$ and write $A \prec \boldsymbol{K} B$ if for every $B^{\prime} \in \boldsymbol{K}_{-1}$ with $B^{\prime} \subseteq B, \delta\left(B^{\prime} / B^{\prime} \wedge A\right) \geq 0$.

2. We denote by $\overline{\boldsymbol{K}}_{0}$ the set of $A \in \overline{\boldsymbol{K}}_{-1}$ which have $\delta\left(A^{\prime}\right) \geq 0$ for each $A^{\prime} \subseteq A$ and by $\boldsymbol{K}_{0}$ those in $\overline{\boldsymbol{K}}_{0}$ which are finitely generated. $T_{0}$ denotes the theory of $\overline{\boldsymbol{K}}_{0}$.

Now it is easy to show

Theorem 4.1.3 Any class $(\boldsymbol{K}, \prec \boldsymbol{K})$ where $\prec \boldsymbol{K}$ is defined from a $\delta$-function from a class $\overline{\boldsymbol{K}}_{-1}$ as in Definition 4.1.2 and that is closed under unions of increasing chains is an Abstract Elementary Class. If it has countable similarity type then the Löwenheim number is $\aleph_{0}$ (Definition 2.0.8).

Since $\prec \boldsymbol{K}$ is imposed by $\delta$, the following properties hold, which are more special than AEC's in general or even the analysis of generic models in [33].

- A6. If $A, B, C \in K(N), A \prec_{K} C, B \subseteq C$, then $A \cap B \prec_{K} B$.

We can restrict to $\overline{\boldsymbol{K}}_{0}$ to obtain: 
- A7. $\emptyset \in \overline{\boldsymbol{K}}_{0}$ and $\emptyset \prec_{\boldsymbol{K}} A$ for all $A \in \overline{\boldsymbol{K}}_{0}$.

A predimension $\delta$ also allows us to construct a combinatorial geometry.

Definition 4.1.4 1. For $M \in \overline{\boldsymbol{K}}_{0}, A \subseteq M, A \in \boldsymbol{K}_{0}, d_{M}(A)=\inf \left\{\delta(B): A \subset B \subseteq M, B \in \boldsymbol{K}_{0}\right\}$.

2. For $A, b$ contained $M, b \in \operatorname{cl}(A)$ if $d_{M}(b A)=d_{M}(A)$.

Naturally we can extend to closures of sets, which are not finitely generated, by imposing finite character.

Lemma 4.1.5 1. The closure system defined in Definition 4.1 .4 is monotone and idempotent as in Assumption 3.1.1.

2. If, in addition $\delta$ is a predimension (integer range) and for any finite $X, d_{M}(X) \leq|X|$ then the closure system satisfies exchange, Assumption 3.1.5.

Definition 4.1.6 1. The pair $(\boldsymbol{K}, \prec \boldsymbol{K})$ has the amalgamation property if for $N, M \in \boldsymbol{K}$ with $A \prec \boldsymbol{K} M, N$, there exists $N_{1} \in \boldsymbol{K}$ and embeddings of $M, N$ as strong submodels of $N_{1}$, which agree on $A$. It has the joint embedding property if any $N, M$ have a common strong extension.

2. The model $M$ is $\kappa$ - $(\boldsymbol{K}, \prec \boldsymbol{K})$-homogeneous (or rich [39]) if $A \prec \boldsymbol{K} M, A \prec \boldsymbol{K} B \in \boldsymbol{K}$ and $|B| \leq \kappa$ implies there exists $B^{\prime} \prec{ }_{K} M$ such that $B \cong_{A} B^{\prime}$.

3. The generic model $\mathbb{G}$ is the unique countable model $\aleph_{0^{-}}(\boldsymbol{K}, \prec \boldsymbol{K})$-homogeneous which is a union of a chain of finitely generated models each of which is a strong extension of its predecessor.

Now the standard arguments show:

Theorem 4.1.7 1 . If a class $\left(\boldsymbol{K}_{0}, \prec \boldsymbol{K}\right)$ has the amalgamation property and the joint embedding property then there is a countable generic structure $\mathbb{G}$.

2. Moreover, for every $\kappa$, there is a structure $M_{\kappa}$ which is $\kappa-(\boldsymbol{K}, \prec \boldsymbol{K})$-homogeneous.

Note that we have amalgamation over models, not over sets and the homogeneity is with respect to strong substructures, not sequential homogeneity. To determine such properties of the generic model as $\omega$-saturation and stability class requires that we introduce a second notion of closure.

Definition 4.1.8 1. Let $A \subseteq M \in \boldsymbol{K}$. The intrinsic(or self-sufficient) closure of $A$ in $M$, denoted icl $\mathrm{M}_{\mathrm{M}}(\mathrm{A})$ is the unique minimal $N$ such that: $A \subseteq N, N \in \boldsymbol{K}, N \prec_{\boldsymbol{K}} M$.

2. We say $B$ is a minimal intrinsic extension of $A$ if $\delta(B / A)<0$ but $\delta\left(B^{\prime} / A\right) \geq 0$ for every $B$ with $B \supset B^{\prime} \supseteq A$.

It is easy to check that icl $\mathrm{M}_{\mathrm{M}}(\mathrm{A})$ can also be constructed by interatively taking minimal intrinsic extensions. It is crucial that this notion be definable (in roughly the same sense one says Morley rank is definable). For example, this is necessary to guarantee that $\overline{\boldsymbol{K}}_{0}$ is axiomatizable. We say $\boldsymbol{K}$ has $\delta$-formulas for minimal intrinsic extensions if for each pair $(B, A)$ with $B$ minimal intrinsic over $A$, there is a formula $\phi_{A B}(\mathbf{x}, \mathbf{y})$ such that if $\phi_{A B}\left(\mathbf{b}^{\prime}, \boldsymbol{a}^{\prime}\right)$ and $B^{\prime}, A^{\prime}$ are the structures generated $\mathbf{b}^{\prime}, \boldsymbol{a}^{\prime}$ then $\delta\left(B^{\prime} / A^{\prime}\right) \leq \delta(B / A)$ (and some other conditions 
we won't spell out here). The existence of $\delta$ formulas is trivial in the ab initio case [25], routine for bicolored fields [7] and impossible (in full generality) for fields with a distinguished multiplicative subgroup [22, 40].

The following key facts about this notion follow from the definition of $\overline{\boldsymbol{K}}_{0}$ as the class of structures with hereditarily non-negative $\delta$. The key points for 2) are that any minimal intrinsic extension $B$ of $A$ can have only finitely many copies in $M$ and this implies that $\phi_{A B}$ is algebraic and any point in the intrinsic closure arises through finitely many iterations of minimal intrinsic extensions.

Lemma 4.1.9 Suppose $\boldsymbol{K}$ has $\delta$-formulas for minimal intrinsic extensions. Let $A \subseteq M \in \overline{\boldsymbol{K}}_{0}$.

1. If $A$ is finitely generated then $\mathrm{icl}_{\mathrm{M}}(\mathrm{A})$ is finitely generated.

2. $\operatorname{icl}_{\mathrm{M}}(\mathrm{A}) \subseteq \operatorname{acl}_{\mathrm{M}}(\mathrm{A})$.

Definition 4.1.10 $\boldsymbol{K}$ is set-determined if for every $X \subset A \in \boldsymbol{K}$ there is a) a minimal $X^{\prime}$ contained in $A$ with $X^{\prime} \in \boldsymbol{K}$ and $\left.b\right) \operatorname{tp}(X / \emptyset)$ determines $\operatorname{tp}\left(X^{\prime} / \emptyset\right)$.

In either the ab initio case (see Section 4.2) or if $\boldsymbol{K}$ arises by naming a subset of an algebraically closed field, the class $\boldsymbol{K}$ is set-determined.

With these observations we see immediately:

Lemma 4.1.11 Suppose $\boldsymbol{K}$ is set-determined. The countable generic model $\mathbb{G}$ is $\aleph_{0}$-homogeneous. More generally, a $\kappa$ - $(\boldsymbol{K}, \prec \boldsymbol{K})$-homogeneous $M$ is $\kappa$-set homogeneous

Proof. Let $\boldsymbol{a}$ and $\mathbf{b}$ be sequences of length less than $\kappa$ from $M$ which realize the same first order type and let $c \in M$; we must find $d$ so that $\boldsymbol{a} c$ and $\mathbf{b} d$ also realize the same type. By Lemma $4.1 .92, \operatorname{icl}_{\mathbb{G}}(\boldsymbol{a}) \cong \operatorname{icl}_{\mathbb{G}}(\mathbf{b})$ and they are finitely generated in the countable case (have cardinality $<\kappa$ in the uncountable case.) Since $\boldsymbol{K}$ is setdetermined, we can replace these intrinsic closures by the models they generate. So there is an automorphism $\alpha$ of $G$ taking one to the other and $\alpha(c)$ is the required $d$.

In [60], Zilber remarks that the categoricity of a structure depends on its 'dimension' and 'homogeneity'. Our countable model $\mathbb{G}$ is a candidate; the dimension theory is given by the geometry and it is homogeneous if $\boldsymbol{K}$ is set-determined. We describe below a variant of this construction to construct a quasiminimal excellent class which is not homogeneous.

We will discuss two strategies for producing categoricity via the Hrushovski construction: the first order strategy and the quasiminimal excellent strategy.

The first order strategy aims to show that the structure $\mathbb{G}$ is strongly minimal in the geometry ( $\alpha=1$ see 4.1.1) case and at least $\aleph_{1}$-categorical otherwise. (It is often almost strongly minimal; see [6]. When $\alpha=1$, for every $M$ and for every singleton $a, d_{M}(a) \leq 1$. The key idea to demand that if $d_{M}(a)=0$, then $a$ is algebraic-thus get strong minimality- by controlling the primitive extensions:

Definition 4.1.12 Let $A, B \in \boldsymbol{K}_{0}$. We say $A$ is primitive over $B$ if $\delta(A / B)=0$ and for any $A^{\prime}$ with $B \subset$ $A^{\prime} \subset A, \delta\left(A / A^{\prime}\right)<0$.

In the $a b$ initio case one needs to also minimize the base; in the bicolored field case this falls out from the general theory of canonical bases. 
The following description (accurate in the ab initio case) oversimplifies the statement in e.g., the bicolored field case, but expresses the spirit of the argument. Suppose $A / B \in \boldsymbol{K}_{0}$ is primitive, let $M$ be $\left(\boldsymbol{K}_{0}, \prec \boldsymbol{K}\right)$ homogeneous and let $\chi_{M}(A / B)$ denote the number of copies of $A$ over $B$ in $M$. To guarantee $\aleph_{1}$-categoricity of the generic, one studies the subclass $\boldsymbol{K}^{\mu}$ of $\boldsymbol{K}_{0}$ where each for each primitive $A / B$,

$$
\chi_{M}(A / B) \leq \mu(A / B)
$$

for a given function $\mu$ from primitive pairs into the integers.

If the generic model for $\boldsymbol{K}^{\mu}$ is $\omega$-saturated, categoricity follows easily. Baldwin and Holland [7] provide a sufficient condition for the $\omega$-saturation of the generic. Another approach is to show that the types which are directly controlled by the geometry do in fact determine the entire theory. Hrushovski [24] summarized the goal of this strategy as the production of a Robinson theory - essentially a universal theory with the amalgamation property. (Hrushovski gave a syntactic condition equivalent to amalgamation by [2].) Then [24] proves that (on the existentially closed models of a Robinson theory) all existential formulas are equivalent to a (possibly infinite) disjunction of quantifier-free formulas. Definition 4.1 .13 makes the connection with $(\boldsymbol{K}, \prec \boldsymbol{K})$. See also $[26]$.

Definition 4.1.13 Assume $\boldsymbol{K}$ has $\delta$-formulas for minimal intrinsic extensions over subsets. Form the language $L^{+}$by adding a relation symbol $R_{A B}(\mathbf{x})$ for each pair $(A, B)$ where $B$ is a minimal intrinsic extension of $A$. For any of our theories, $T^{0}, T_{n a t}^{0}$ is the $L^{+}$-theory extending $T^{0}$ which asserts:

$$
\left[\exists \mathbf{y} \phi_{A B}(\mathbf{x}, \mathbf{y})\right] \leftrightarrow R_{A B}(\mathbf{x}) .
$$

We denote the natural expansion of an L-structure $N$ to $L^{+}$by $N^{+}$and the collection of expansions of models in a class $\boldsymbol{K}$ by $\boldsymbol{K}^{+}$.

If the theory of the existentially closed in $L^{+}$models of $\boldsymbol{K}^{+}$is first order axiomatizable then it is quantifier eliminable. Thus one technique for determining $\omega$-stability (or strong minimality) is just to study the quantifier free $L^{+}$-types. In fact, as we briefly describe in the next subsection most of the published work uses two other techniques.

\subsection{The first order case}

In the first order situation, the first step is:

I. Show $\boldsymbol{K}_{0}=\{A: \delta(A) \geq 0\}$ is first order axiomatizable.

Now the aim is to construct a complete first order theory. One approach is to show

II. the generic model is saturated and take its theory.

A weaker approach is to show one of

IIIa Show $\boldsymbol{K}^{e c}$, the class of existentially closed for $\boldsymbol{K}_{0} L$-structures is first order.

IIIb Show $\boldsymbol{K}^{e c,+}$, the class of existentially closed for $\boldsymbol{K}_{0} L^{+}$-structures is first order.

IIIc Show that the class of $\boldsymbol{K}_{0^{-}} \prec \boldsymbol{K}$ homogeneous universal models is first order axiomatizable.

If the generic model is saturated then IIIb holds. But there are cases where IIIb holds but the generic is not saturated. (e.g. the Shelah-Spencer random graph [9]). And [1] provides a 'toy' example where $\delta$ maps into the integers but the generic is not $\omega$-saturated. 
Poizat has introduced the study of an intermediate stage; construct various expansions of fields with infinite rank by the Hrushovski construction $([39,40])$. This exercise helps to illuminate the situation in a simpler case than actually finding $\aleph_{1}$-categorical structures as in $[25,5,7,58]$. We briefly compare the $\omega$-stable case, $\boldsymbol{K}$, and the $\aleph_{1}$-categorical case, $\boldsymbol{K}^{\mu}$, in three situations.

Ab initio Hrushovski proved $\aleph_{1}$-categoricity of $\boldsymbol{K}^{\mu}$ in the seminal [25]. Poizat [14] simplified the argument to $\boldsymbol{K}$ where the structure constructed has infinite rank. Holland [23] proved that the strongly minimal sets were model complete. Baldwin and Holland [6] have shown that Baldwin's projective plane [5] is model complete after adding some constants.

Bicolored fields: Expand $\mathbb{C}$ by an infinite unary predicate. For $\boldsymbol{K}$, IIIc and II are fairly straightforward [39]. Note that IIIa fails although IIIb follows from II. The harder II for $\boldsymbol{K}^{\mu}$ is done by Baldwin-Holland [7]. For this, it is essential that the function $\mu$ be finite-to-one. Baldwin and Holland [6] have shown that the infinite rank bicolored field is not model complete, while the finite rank bicolored fields are. It is easy to check that bicolored fields are set-determined. So we get a homogeneous model even if $\mu$ is not finite to one. It is shown in [7] that the generic need not be saturated.

Bad fields: Expand $\mathbb{C}$ by naming an infinite (torsion-free) subgroup of the multiplicative group. I is done for $\boldsymbol{K}$ by Poizat [40]/independently by Baldwin-Holland (Marker) (unpublished), using the Zilber-Hrushovski true CIT (see Theorem 5.1.1). IIIc and II are sketched by Poizat [40]. Holland has independent work [22] which yields a complete proof. For $\boldsymbol{K}^{\mu}$, much remains open although I follows.

\subsection{Analytic models of the Hrushovski construction}

Zilber has suggested the following problem which we noted in the first paragraph of this paper.

Thesis. [Zilber] Hrushovski models can be obtained as pseudoanalytic structures (i.e as reducts of 'analytic' expansions of the complex numbers).

One viewpoint of this thesis is that the notion of pseudo-analytic structure (analytic expansion of the complex field) is a generalization of the notion of definable in a field, which will make the Zilber's trichotomy conjecture true (i.e. 'psuedoanalytic' replaces 'field-like structure' in stating the trichotomy). There are two papers by Wilkie [55] and Zilber [56] in the Proceedings of Paris, 2000; Zilber's expounds the thesis and Wilkie's makes serious steps towards proving one example has such a representation.

The justification for this conjecture is primarily philosophical: 'natural = canonical'. Again, canonical is read as categorical in some reasonable syntax. Complex exponentiation is natural; ergo it must be canonical (see Subsection 5. The Hrushovski constructions yield categorical objects, thus they must be representable in a natural object.

The full conjecture is false as stated. It is certainly impossible to realize strongly minimal sets as structures whose definable relations are analytic subsets of the complex numbers. (Analytic functions cannot be finite-toone.) Zilber has some notions on weakening 'analytic'. Some is reported in [38]. Roughly, the idea is that the strongly minimal structure can be found as a restriction of an analytic structure to a collection of infinitesimal neighborhoods.

There is progress on modeling $\omega$-stable examples. In particular, there is one fully worked out exemplar of this conjecture; obtained by adding a generic unary function to the complex numbers. Consider the language $L:+, \cdot, 0,1, H(x)$. Koiran [32], building on Wilkie [55] defines the limit theory of generic polynomials as the model completion of the class $\boldsymbol{K}_{0}$ arising from

$$
\delta\left(x_{1}, \ldots x_{n}\right)=d_{f}\left(x_{1}, \ldots x_{n}, H\left(x_{1}\right), \ldots H\left(x_{n}\right)\right)-\left|\left(x_{1}, \ldots x_{n}\right)\right| .
$$

He proves: 
Theorem 4.3.1 (Koiran) The limit theory of generic polynomials has a model $(\mathbb{C},+, \cdot, f)$ where $f$ is an analytic function.

The function $f$ is

$$
f(x)=\Sigma_{i=1}^{\infty} \frac{x^{i}}{a_{i}}
$$

where for every $\ell \geq 1,\left|a_{i+1}\right| \geq\left|a_{i}\right|^{\ell}$, for all sufficiently large $i$. Intuitively, the functions obtained by truncating $f$ at $x^{n}$ approximate generic degree $n$ polynomials. From the general theory we see the theory is first order $\omega$-stable. Zilber pointed out that the analytic model $M$ is quasi-minimal excellent and so the $L_{\omega_{1}, \omega}(Q)$ theory of $M$ is categorical. Thus among the models with power $\aleph_{1}$, we can choose with $L(Q)$ one which is canonical (categorical); this model has many small definable sets. A priori, the saturated model might seem more canonical. But, an $\aleph_{1}$-saturated model is only categorical in $L_{\omega_{1}, \omega}(Q)$ if it is first order categorical. (By Theorem 2.0.3, the model of cardinality $\aleph_{1}$ is small. The categoricity gives that every $\omega$-saturated model of $T=\operatorname{Th}(M)$ is saturated. Thus $T$ is $\omega$-stable. If $T$ is uni-dimensional, we are done. If not, $T$ has non-isomorphic $\omega$-saturated models in every uncountable power $[44,4]$.

\section{Complex Exponentiation $(\mathbb{C},+, \cdot, \exp )$}

The most ambitious aim of the pseudo-analytic model program is to realize $(\mathbb{C},+, \cdot, \exp )$ as a model of an $L_{\omega_{1}, \omega}$-sentence discovered by the Hrushovski construction. This program has two parts.

A. Model theory: Using a Hrushovski like dimension function, expand $(\mathbb{C},+, \cdot)$ by a unary function $f$ which behaves like exponentiation. Prove that the theory $\Sigma$ of $(\mathbb{C},+, \cdot, f)$ in an appropriate logic is well behaved. (Two options for this theory are discussed in Subsections 5.2 and 5.3

B. Prove $(\mathbb{C},+, \cdot, \exp )$ is a model of the sentence $\Sigma$ found in Objective A.

Zilber's work on this program involves several algebraic advances which we recount in 5.1. In the last two subsections we report two versions of the program; the first considers raising to real powers, the second full complex exponentiation. In the entire section we restrict to characteristic 0 .

\subsection{The necessary algebra}

There are several algebraic results/conjectures which are needed for this program.

Theorem 5.1.1 $\quad$ 1. Zilber's conjecture on Intersection of tori (CIT) has two forms. Let $W \subseteq C^{n}$ be an algebraic variety defined over $\mathbb{Q}, T \subseteq\left(C^{*}\right)^{n}$ a torus, and $S$ an infinite irreducible component of $W \cap T$. We say $S$ is an atypical component if

$$
\operatorname{dim} S>\operatorname{dim} W+\operatorname{dim} T-n
$$

(a) true CIT: This is proved in [59]:

Theorem 5.1.2 [Zil'ber] Given a variety $W \subseteq \mathbb{C}^{n+k}$ defined over $\mathbb{Q}$, there is a finite set $A$ of nonzero elements of $\mathbb{Z}^{n}$ such that given any coset $T \subseteq\left(\mathbb{C}^{*}\right)^{n}$ of a torus and any $\mathbf{b} \in \mathbb{C}^{k}$, if $S$ is an atypical component of $W(\mathbf{b})$ (where $W(\mathbf{b})=\{\boldsymbol{a} \subseteq \mathbb{C}: \mathbf{a} \mathbf{b} \in W\}$ ) then for some $\mathbf{m} \in A$ and some $\gamma$ from $\mathbb{C}$, every element of $S$ satisfies $\mathbf{x}^{\mathbf{m}}=\gamma$. 
(b) full CIT:

Conjecture 5.1.3 For any variety $W \subseteq \mathbb{C}^{n}$ defined over $\mathbb{Q}$, there is a finite collection $\tau(W)=$ $\left\{T_{1}, \ldots T_{k}\right\}$ of proper basic tori in $\left(C^{*}\right)^{n}$ such that for any proper basic torus $T \subseteq\left(C^{*}\right)^{n}$ and any atypical component of $W \cap T$,

$$
S \subseteq T_{i} \text { for some } T_{i} \in \tau(W) .
$$

2. The thumbtack lemma, which says that divisibly closed multiplicatively closed subgroups are finitely determined, Theorem 3.2.5.

3. Schanuel's conjecture: If $x_{1}, \ldots x_{n}$ are $\mathbb{Q}$-linearly independent complex numbers then $x_{1}, \ldots x_{n}, e^{x_{1}}, \ldots e^{x_{n}}$ has transcendence degree at least $n$ over $\mathbb{Q}$.

The full CIT can be seen [59] as a generalization of the Mordell-Lang and Manin-Mumford conjectures; we see a model theoretic consequence below.

Objective A relies on the thumbtack lemma to prove excellence as in Subsection 3.2. True CIT can be used to make some axioms first order. That application is not strictly necessary for the problem here; similar applications are essential for approaching the construction of a first order finite rank bad field. Objective B, describing complex exponentiation, requires Schanuel's conjecture and a proof that complex exponentation satisfies the strong exponential closure axioms (described below).

\subsection{Raising to powers}

In [61] Zilber considers structures: $(D, \exp , R)$ where $D$ is an infinite dimensional vector space over a fixed countable field $K$ of characteristic $0, R$ is a field of characteristic 0 , exp is a homomorphism of the additive group of $D$ onto the multiplicative group $R^{*}$ of the field.

The formula $(\exists z) z=\exp (z) \wedge y=\exp (a \cdot z)$ with $D$ and $R$ both the complexes defines the multifunction $y=z^{a}$.

In this situation Objective A is approached by another first order example of the Hrushovski construction. The appropriate rank is given by:

$$
\delta(X)=l d_{K}(X)+d_{f}(\operatorname{Ex}(X))-l d_{Q}(X)
$$

Here Ex is a unary function that is being axiomatized. Zilber gets positive solutions for steps I (using true CIT) and IIIb of the first order strategy and concludes that every completion of the theory is superstable. This gives him an approximation to objective A), superstability rather than categoricity. Using Schanuel and full CIT, and now interpreting Ex as exp, complex exponentiation, he gets the following instance of B):

Theorem 5.2.1 (Zilber) Assume full CIT and SchC. The first order theory of the complex numbers with raising to all real powers allows quantifier elimination in an appropriate language and is superstable.

\subsection{Pseudo-exponentiation}

A pseudo-exponential is unary function from the additive group of a field to the multiplicative group that satisfies certain conjectural properties of complex exponentiation. In this section we outline the argument concerning complex exponentiation from [58] which obtains objective A outright and formulates precise algebraic conjectures sufficient for objective B. Section 3.2 concerns quasiminimal excellent classes without the Hrushovski 
construction; Sections 4.2 and 5.2 concerns the first order Hrushovski construction. Here the two methods are joined.

The quasiminimal excellent strategy: Prove some $L_{\omega_{1}, \omega}$-sentence $\Sigma$, satisfied by $(\mathbb{C},+, \cdot, f)$ is quasiminimal excellent and has quantifier elimination. We follow Zilber and describe $\Sigma$ by successively presenting classes, denoted by $\mathcal{E}$ with various decorations, culminating in $\mathcal{E} C_{s t}^{*}$ which is the quasiminimal excellent class.

Fix the language $L$ to contain $+,\left\{\frac{1}{m}: m \in \omega\right\}, E,\{V(\mathbf{x}): \mathrm{V}$ a variety $\}$.

Assumption 5.3.1 Let $\mathcal{E}$ be the class of L-structures $F$ where $F$ is an algebraically closed field, and $E$ is the graph of a surjective map exp from $F$ to $F^{*}$, which is a homomorphism between the additive and multiplicative group.

Note that we have the graph of multiplication but not the multiplication function; this allows to consider partial maps which approximate our eventual exp. Denote by $s u b \mathcal{E}$ the class of all substructures $A$ of members of $\mathcal{E}$ such the domain $\mathbf{D}_{A}$ of exp is closed under addition and multiplication by rationals.

This construction varies from the first order case in several respects. One technical innovation is that the dimension function $\delta$ is defined relative to its ambient structure $A$. For $X \subset_{\omega} A$,

$$
\delta_{A}(X)=d_{f}(X \cup \operatorname{span}(\exp X))-l d(X)
$$

More important, the actual 'amalgamation class' is restricted in two ways.

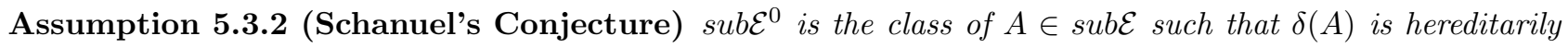
nonnegative.

The assertion that

$$
\delta_{A}(X)=d_{f}(X \cup \operatorname{span}(\exp X))-l d(X) \geq 0
$$

amounts for $A=\mathbb{C}$ and $X$ linearly independent to the Schanuel conjecture. At this point, this is only a requirement on an abstract function exp. A priori the axiom can be expressed in $L_{\omega_{1}, \omega}$; using the HollandPoizat-Zilber variant on true CIT, the axiom can be made first order.

For $A \in s u b \mathcal{E}$, the kernel of the exponential map, $\operatorname{ker}_{A}=\{a: a \in A \wedge \exp (a)=1\}$. If $\operatorname{ker}_{A}$ is 1 -generated we say it is standard; if $\mathbf{D}_{A} / \operatorname{ker}_{A}$, as a subgroup of $k^{*}$ for algebraically closed $k^{*}$, contains all the torsion points we say $A$ has full kernel. Now

Assumption 5.3.3 (Z-standard) subE $_{\text {st }}^{0}$ is the class of $A \in$ subE $^{0}$ such that $\operatorname{ker}_{A}$ is both standard and full.

Stating this condition is the first of several uses of $L_{\omega_{1}, \omega}$. The requirement that the kernel of the function $f$ is always $Z$ leads to the failure of homogeneity.

Assumption 5.3.4 (Existential Closure) Any free and non-overdetermined irreducible system of polynomial equations

$$
P\left(x_{1}, \ldots x_{n}, y_{1}, \ldots y_{n}\right)
$$

has a generic solution satisfying

$$
y_{i}=f\left(x_{i}\right) .
$$

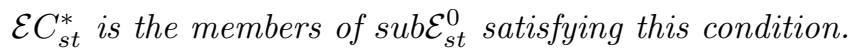


To demand a generic solution, $L_{\omega_{1}, \omega}$ is needed.

What does non-overdetermined mean? It means the variety defined by $P$ is absolutely free of additive dependencies (of multiplicative dependencies) and normal. Let $V$ be a variety in $2 n$ variables and let $\operatorname{pr}_{\mathbf{x}} V$ denote the projection on $\mathbf{x}, \operatorname{pr}_{\mathbf{y}} V$ the projection on $\mathbf{y}$. A variety $V$ contained in $F^{2 n}$, which is definable over $A \cup f(C) \cup \operatorname{ker}(f)$, is absolutely free of additive dependencies (of multiplicative dependencies) if for any generic realization $\boldsymbol{a}$ of $\operatorname{pr}_{\mathbf{x}} V$ is additively (multiplicatively) linearly independent over $\operatorname{acl}(A)$. $V$ is normal if (very roughly) for $k \leq n$, any 'linear image' of $V$ in $F^{2 k}$ has dimension at least $k$.

We say $F$ is strongly exponentially algebraically closed if for any exp-irreducible, additively and multiplicatively free, normal $V$ defined over a finite $C \subset F$, there is a generic over $C$ realization of $V$ in $F$. So we can rephrase Assumption 5.3.4 as $\mathcal{E} C_{s t}^{*}$ is the class of strongly exponentially algebraically closed structures.

Define a closure operation $\mathrm{cl}_{F}(A)$ from $\delta$ exactly as in Definition 4.1.4.

Theorem 5.3.5 $A \rightarrow \operatorname{cl}_{F}(A)$ in $F \in \mathcal{E} C_{\text {st }}$ is a closure operation (with exchange) and for any $A, \operatorname{cl}(A)$ is a strongly exponentially algebraically closed subset of $F$.

Finally,

Assumption 5.3.6 (Countable Closure) The closure of any countable subset is countable.

Now we obtain objective A.

Theorem 5.3.7 $\mathcal{E} C_{s t}^{*}$ (see 5.3.4) is $L_{\omega_{1}, \omega}$-axiomatizable and in fact quasiminimal excellent.

The members of $\mathcal{E} C_{s t}^{*}$ with countable closure are categorical in all uncountable powers. This class is $L_{\omega_{1}, \omega}(Q)$ axiomatizable.

The argument for this is similar to most Hrushovski constructions but requires several new algebraic-modeltheoretic definablity results: The set of $\mathbf{z}$ such that $V(\mathbf{x}, \mathbf{y}, \mathbf{z})$ satisfies any of the following conditions is first order definable: exp-irreducible, absolutely additively free, absolutely multiplicatively free, normal. This key fact depends on true CIT and the refining of it proved independently by Holland, Poizat, and Zilber.

Theorem 5.3.7 concludes Objective A and Objective B is given by:

Theorem 5.3.8 If the Schanuel conjecture holds in $\mathbb{C}$ and if the strong exponential closure axioms hold in $\mathbb{C}$, then $(\mathbb{C},+, \cdot, \exp ) \in \mathcal{E} C_{s t}^{*}$. ( $\left.\mathbb{C},+, \cdot, \exp \right)$ has the countable closure property.

The hypothesis of this theorem is a research program. Work on Schanuel's conjecture has continued for fifty years; Zilber's existential closure condition yield new and interesting number theoretic problems. There are intimate connections with Mordell-Lang.

Note that while the first four conditions yield an AEC, there is no reason to think that countable closures is preserved by unions.

This program leads to a more general question. Are there general conditions under which an AEC induced from a rank $\delta$ as in Definition 4.1.4 must be excellent? 


\section{Summary}

The work discussed in this paper ties together most of the model theory of the last 50 years. Shelah's attempts to generalize the Morley theorem to infinitary logic yield a number of partial results. In particular, the notion of excellence is isolated as a key to the structure theory of uncountable models while the notion of Abstract Elementary Classes arises naturally in attempting to prove the categoricity theorem for $L_{\omega_{1}, \omega}(Q)$. From another direction Zilber attempts to identify canonical mathematical structures as those whose theory (in an appropriate logic) is categorical in all powers. The trichotomy conjecture is refuted by Hrushovski, who introduces a special kind of Abstract Elementary Class. Zilber's use of these techniques to investigate complex exponentiation yields not only exciting model theory but new results and conjectures in algebraic geometry.

\section{References}

[1] Roman Aref'ev, J.T. Baldwin, and M. Mazzucco. $\delta$-invariant amalgamation classes. Journal of Symbolic Logic, 64:1743-1750, 1999.

[2] P.D. Bacsich and Rowlands Hughes D. Syntactic characterisations of amalgamation, convexity, and related properties. J. Symbolic Logic, 39:433-51, 1974.

[3] John T. Baldwin. Rank and homogeneous structures. In Katrin Tent, editor, Tits Buildings and the Theory of Groups Wurzburg Sept 14-17 2000. Cambridge University Press, 2002.

[4] J.T. Baldwin. Fundamentals of Stability Theory. Springer-Verlag, 1988.

[5] J.T. Baldwin. An almost strongly minimal non-desarguesian projective plane. Transactions of the American Mathematical Society, 342:695-711, 1994.

[6] J.T. Baldwin and K. Holland. Constructing $\omega$-stable structures: Existential closure. Annals of Pure and Applied Logic, 200? to appear.

[7] J.T. Baldwin and K. Holland. Constructing $\omega$-stable structures: Rank 2 fields. Journal of Symbolic Logic, 65:371-391, 2000.

[8] J.T. Baldwin and K. Holland. Constructing $\omega$-stable structures: Rank k. Notre Dame Journal of Formal logic, 2001. to appear.

[9] J.T. Baldwin and S. Shelah. Randomness and semigenericity. Transactions of the American Mathematical Society, 349:1359-1376, 1997.

[10] J.T. Baldwin and Niandong Shi. Stable generic structures. Annals of Pure and Applied Logic, 79:1-35, 1996.

[11] S. Buechler and O. Lessmann. Simple homogeneous models. Journal of the American Mathematical Society, 16:91-121, 2003.

[12] C. C. Chang. Some remarks on the model theory of infinitary languages. In J. Barwise, editor, The syntax and semantics of infinitary languages, pages 36-64. Springer-Verlag, 1968. LNM 72.

[13] R. Frä̈ssé. Sur quelques classifications des systèmes de relations. Publ. Sci. Univ. Algeria Sèr. A, 1:35-182, 1954.

[14] John B. Goode. Hrushovski's Geometries. In Helmut Wolter Bernd Dahn, editor, Proceedings of 7th Easter Conference on Model Theory, pages 106-118, 1989. 
[15] Rami Grossberg. Classification theory for non-elementary classes. In Yi Zhang, editor, Logic and Algebra, pages 165-204. AMS, 2002. Contemporary Mathematics 302.

[16] Rami Grossberg and Bradd Hart. The classification theory of excellent classes. Journal of Symbolic Logic, 54:1359-1381, 1989).

[17] Rami Grossberg and Olivier Lessmann. Shelah's stability spectrum and homogeneity spectrum in finite diagrams. Archive for Mathematical Logic, 41:1-31, 2002).

[18] Bradd Hart and Saharon Shelah. Categoricity over $P$ for first order $t$ or categoricity for $\phi \in \mathrm{l}_{\omega_{1} \omega}$ can stop at $\aleph_{k}$ while holding for $\aleph_{0}, \cdots, \aleph_{k-1}$. Israel Journal of Mathematics, 70:219-235, 1990.

[19] W. Henson and Jose Iovino. Ultraproducts in analysis. In Analysis and Logic, volume 262 of London Mathematical Society Lecture Notes, pages 1-115. Cambridge University Press, 2002.

[20] B. Herwig. Weight $\omega$ in stable theories with few types. J. Symbolic Logic, 60:353-373, 1995.

[21] W. Hodges. Model Theory. Cambridge University Press, 1993.

[22] Kitty Holland. Transcendence degree and group rank. preprint.

[23] Kitty Holland. Model completeness of the new strongly minimal sets. J. Symbolic Logic, 64:946-962, 1999.

[24] E. Hrushovski. Simplicity and the Lascar group. preprint.

[25] E. Hrushovski. A new strongly minimal set. Annals of Pure and Applied Logic, 62:147-166, 1993.

[26] T. Hyttinen. Canonical finite diagrams and quantifier elimination. Mathematical Logic Quarterly, 48:533$554,2002$.

[27] T. Hyttinen and S. Shelah. Strong splitting in stable homogeneous models. Annals of Pure and Applied Logic, 103:201-228, 2000.

[28] B. Jónsson. Universal relational systems. Mathematica Scandinavica, 4:193-208, 1956.

[29] B. Jónsson. Homogeneous universal relational systems. Mathematica Scandinavica, 8:137-142, 1960.

[30] H.J. Keisler. Logic with quantifier "there exists uncountably many". Annals of Math. Logic, 1:1-93, 1970.

[31] H.J Keisler. Model theory for Infinitary Logic. North-Holland, 1971.

[32] P. Koiran. The theory of liouville functions. see webpage, 2002.

[33] D.W. Kueker and C. Laskowski. On generic structures. Notre Dame Journal of Formal Logic, 33:175-183, 1992.

[34] Olivier Lessmann. Notes on excellent classes. preprint.

[35] Olivier Lessmann. Homogeneous model theory: Existence and categoricity. Contemporary Mathematics $302,2002$.

[36] L. Marcus. A minimal prime model with an infinite set of indiscernibles. Israel Journal of Mathematics, 11:180-183, 1972.

[37] M. Morley and R.L. Vaught. Homogeneous universal models. Mathematica Scandinavica, 11:37-57, 1962.

[38] N. Peatfield and B. Zilber. Analytic Zariski structures and the Hrushovski construction. preprint, 2003.

[39] Bruno Poizat. Le carré de l'egalité. The Journal of Symbolic Logic, 64:1339-1356, 1999. 
[40] Bruno Poizat. L'egalité au cube. The Journal of Symbolic Logic, 66:1647-1676, 2001.

[41] S. Shelah. Finite diagrams stable in power. Annals of Mathematical Logic, 2:69-118, 1970.

[42] S. Shelah. Categoricity in $\aleph_{1}$ of sentences in $L_{\omega_{1}, \omega}(Q)$. Israel Journal of Mathematics, 20:127-148, 1975. paper 48 .

[43] S. Shelah. The lazy model-theoretician's guide to stability. Logique et Analyse, 18:241-308, 1975.

[44] S. Shelah. Classification Theory and the Number of Nonisomorphic Models. North-Holland, 1978.

[45] S. Shelah. Classification theory for nonelementary classes. I. the number of uncountable models of $\psi \in L_{\omega_{1} \omega}$ part A. Israel Journal of Mathematics, 46:3:212-240, 1983. paper 87a.

[46] S. Shelah. Classification theory for nonelementary classes. I. the number of uncountable models of $\psi \in L_{\omega_{1} \omega}$ part B. Israel Journal of Mathematics, 46;3:241-271, 1983. paper 87b.

[47] S. Shelah. Categoricity of abstract elementary classes: going up inductive step. preprint 600, $200 ?$

[48] S. Shelah. Toward classification theory of good $\lambda$ frames and abstract elementary classes. preprint 705, $200 ?$

[49] S. Shelah. Categoricity of abstract elementary class in two successive cardinals. Israel Journal of Mathematics, 126:29-128, 2001. paper 576.

[50] Saharon Shelah. Classification of nonelementary classes II, abstract elementary classes. In J.T. Baldwin, editor, Classification theory (Chicago, IL, 1985), pages 419-497. Springer, Berlin, 1987. paper 88: Proceedings of the USA-Israel Conference on Classification Theory, Chicago, December 1985; volume 1292 of Lecture Notes in Mathematics.

[51] Saharon Shelah. Universal classes, part I. In J.T. Baldwin, editor, Classification theory (Chicago, IL, 1985), pages 264-419. Springer, Berlin, 1987. paper 300:Proceedings of the USA-Israel Conference on Classification Theory, Chicago, December 1985; volume 1292 of Lecture Notes in Mathematics.

[52] Saharon Shelah and Andrés Villaveces. Toward categoricity for classes with no maximal models. Annals of Pure and Applied Logic, 97:1-25, 1999. revised version : www.math.rutgers.edu/ ${ }^{\sim}$ shelah.

[53] Monica VanDieren. Categoricity and stability in abstract elementary classes. PhD thesis, Carnegie Mellon University, 2002.

[54] R.L. Vaught. Denumerable models of countable theories. In Infinitistic Methods, Proc. Symp. Foundations of Math., Warsaw, 1959, pages 303-321. Oaństwowe Wydawnictwo Naukowe, Warsaw, 1961.

[55] A. Wilkie. Liouville functions. preprint, 2002.

[56] B.I. Zilber. Analytic and psuedo-analytic structures. In Proceedings Of Logic Colloquium 2000, Paris, $200 ?$ to appear.

[57] B.I. Zilber. Covers of the multiplicative group of an algebraically closed field of characteristic 0. preprint, 2000.

[58] B.I. Zilber. Fields with pseudoexponentiation. preprint, 2000.

[59] B.I. Zilber. Intersecting varieties with tori. preprint, 2000.

[60] B.I. Zilber. A categoricity theorem for quasiminimal excellent classes. preprint, 2002.

[61] B.I. Zilber. Raising to powers in algebraically closed fields. preprint, 2002. 